\title{
Évaluation des prises de décisions de limitations et d'arrêt des thérapeutiques chez les patients décédés aux urgences
}

\section{Assessment of Withholding and Withdrawing Life-Sustaining Treatments of Deceased Patients in an Emergency Department}

\author{
P. Vaittinada Ayar $\cdot$ S. Ayllon-Milla $\cdot$ C. Damas-Perrichet $\cdot$ B. Villoing $\cdot$ B. Doumenc $\cdot$ F. Dumas \\ Reçu le 26 février 2018 ; accepté le 5 juillet 2018 \\ (C) SFMU et Lavoisier SAS 2018
}

Résumé Introduction : Les décisions de limitation et arrêt des thérapeutiques (LAT) sont encadrées par la loi du 22 avril 2005 relative aux droits des malades et à la fin de vie. Au lendemain de sa révision, le 2 février 2016, cette situation reste toujours aussi complexe aux urgences. Notre travail avait pour but d'évaluer la prise de décision des limitations de soins aux urgences.

Matériel et méthode : Il s'agissait d'une étude rétrospective, observationnelle, monocentrique. Le recueil des données a été fait par une relecture des dossiers concernant les patients décédés entre le $1^{\text {er }}$ janvier 2014 et le 5 mai 2015. L'objectif de notre travail était de décrire les modalités de prise de décision de limitations de soins, ainsi que leur adéquation avec la loi. Résultats : Nous avions inclus 91 dossiers de patients décédés. Parmi les 58 dossiers (64 \%) où l'autonomie était retrouvée, elle était très limitée chez 50 patients (Knaus $\mathrm{C}+\mathrm{D})$, soit $86 \%$. La décision était inscrite dans 74 dossiers ( $81 \%$ ), mais détaillée dans 40 seulement (44\%). Soixante-quinze fois $(83 \%)$, la famille a été informée. Dans $70(80 \%)$ des 87 situations où les intervenants étaient identifiés, la procédure collégiale était respectée. Une thérapeutique palliative a été initiée chez 67 des défunts (74\%).

Conclusion : Les décisions de LAT sont encore perfectibles aux urgences pour être en adéquation avec la loi. Des améliorations sont possibles en intégrant des programmes de formations du personnel et des protocoles éthiques d'aide à la décision.

\footnotetext{
P. Vaittinada Ayar $(\bowtie) \cdot$ S. Ayllon-Milla $\cdot$ C. Damas-Perrichet

B. Villoing $\cdot$ B. Doumenc $\cdot$ F. Dumas

Service des urgences, hôpital Cochin,

Assistance publique-Hôpitaux de Paris (AP-HP),

hôpitaux universitaires Paris-Centre,

27, rue du Faubourg-Saint-Jacques, F-75014 Paris, France

e-mail : pvaittinada@gmail.com

F. Dumas

UMR Inserm 970, université Paris-Descartes,

F-75006 Paris, France
}

Mots clés Limitation et arrêt des thérapeutiques · Fin de vie · Soins palliatifs $\cdot$ Urgences

\begin{abstract}
Aim: Withholding life-sustaining treatment (WHLST) and withdrawing life-sustaining treatment (WDLST) decisions are ruled by the act of 22 April 2005 on patients' rights and the end-of-life. After its revision on February 2, 2016, this situation remains complex in the emergency department (ED). The aim of our study is to assess WHLST or WDLST decision at ED.

Procedure: This was a single-center, retrospective, observational study. The data collection was done by chart review of deceased patients between January 1, 2014 and May 5, 2015. The objective of our work was to describe WHLST or WDLST decisions and their adequacy with the law.

Results: We had collected 91 deceased patients' records. Of the 58 cases $(64 \%)$ where autonomy was found, it was very limited in 50 patients (Knaus C $+\mathrm{D}$ ) or $86 \%$. The decision was registered in 74 files (81\%) but detailed only in 40 (44\%). Seventy-five times ( $83 \%$ ) the family was informed. In $70(80 \%)$ of the 87 situations where the participants were identified, the collegiate procedure was respected. Palliative therapy was initiated in 67 patients $(74 \%)$.

Conclusion: WHLST or WDLST decisions are still perfectible at ED to be in adequacy with the law. Improvements are possible by integrating staff training programs and protocols of ethical decision support.
\end{abstract}

Keywords Withholding and withdrawing life-sustaining treatments $\cdot$ End-of-life $\cdot$ Palliative care $\cdot$ Emergency room

\section{Introduction}

Dans un contexte d'augmentation permanente du nombre de passages aux urgences, le pourcentage de décès reste stable et représente 0,1 à $0,2 \%$ de ceux-ci $[1,2]$. Actuellement, près 
de $60 \%$ des décès ont lieu à l'hôpital, dont $16 \%$ aux urgences [2]. Les patients en fin de vie, c'est-à-dire présentant une pathologie chronique évolutive au pronostic à court terme défavorable en termes d'espérance de vie ou d'aggravation de la dépendance [3], représentent 25 à $33 \%$ des décès aux urgences. La loi dite Leonetti du 22 avril 2005 puis la modification de l'article $37 \mathrm{du}$ Code de déontologie en janvier 2010 ont permis d'apporter un cadre législatif précis aux décisions de limitation et d'arrêt des thérapeutiques (LAT). Elle impose une procédure collégiale lors de la prise de décisions de LAT. Si le patient ne peut exprimer sa volonté, la procédure est associée à la consultation de ses directives anticipées (DA), de sa personne de confiance (PDC) ou à défaut de ses proches. La législation insiste également sur l'importance de la traçabilité de la décision.

La révision de cette loi en 2016 vient la renforcer, donnant le droit au patient de limiter ou de refuser les traitements. Elle facilite et généralise la rédaction des DA qui s'imposeront désormais aux médecins. Elles seront inscrites sur sa carte vitale, resteront révisables à tout moment, mais n'auront plus de délai de validité. En pratique, les décisions de LAT sont fréquentes aux urgences. Une étude multicentrique montre que $80 \%$ des décès survenant aux urgences font suite à une décision de LAT [4]. Pourtant, le médecin urgentiste est confronté à de nombreuses difficultés inhérentes à sa fonction pour prendre ces décisions : son isolement, la gestion du flux de patients, le manque de temps, le manque de données sur l'état antérieur du patient et sur ses souhaits, l'impossibilité de joindre le médecin référent la nuit ou le week-end et la charge morale importante $[5,6]$.

Dans cette étude, nous avons voulu décrire les modalités de prise de décision de limitation de soins chez les patients décédés dans un service d'urgence ainsi que leur adéquation avec le cadre légal.

\section{Méthode}

Cette étude rétrospective, observationnelle, descriptive a été réalisée au sein d'un service d'urgence parisien accueillant près de 51000 passages par an. Le recueil des données a été fait dans les dossiers informatisés des patients décédés aux urgences entre le $1^{\text {er }}$ janvier 2014 et le 5 mai 2015, via le logiciel Urqual ${ }^{\circledR}$ (McKesson, San Francisco, États-Unis). Parmi les dossiers des patients décédés, étaient sélectionnés ceux qui comprenaient une décision de LAT, qu'elle ait été explicite ou implicite : soit la notion de LAT apparaissait clairement dans le dossier, soit une démarche de limitation de soins apparaissait dans le dossier, généralement pour les patients en fin de vie pour lesquels les traitements invasifs optimaux ont été jugés inadaptés et n'ont pas été initiés. Les patients exclus étaient ceux en arrêt cardiorespiratoire à l'arrivée ou peu après, ainsi que les patients dont le décès n'était pas « attendu ».

Les données démographiques et médicales des patients ont été recueillies, notamment les antécédents et la préexistence d'une démarche palliative, l'état d'autonomie antérieur, le motif d'admission. L'autonomie des patients a été évaluée au vu du dossier par le calcul du score de Knaus. Des données concernant la prise de décisions de LAT et sa mise en place ont également été collectées : consultation du patient, le cas échéant de ses DA, de sa PDC ou de ses proches, arguments motivant la décision de LAT, intervenants ayant participé à celle-ci, traitements sur lesquels portaient la limitation et les traitements, et les soins de support instaurés. Les traitements invasifs de réanimation étaient ceux retenus par la Société française de médecine d'urgence (SFMU) : une réanimation cardiopulmonaire spécialisée, l'intubation orotrachéale (IOT), la ventilation mécanique, la mise en place de substances vasoactives, le remplissage vasculaire [7]. Les traitements des symptômes d'inconfort étaient ceux préconisés par la Société française d'accompagnement et de soins palliatifs (SFAP) : oxygénothérapie, antalgiques et en particulier morphiniques, traitement anxiolytique et sédation [8].

Les critères d'évaluation de la mise en place de la procédure recherchés dans les dossiers étaient l'information et l'implication des proches et du patient, la recherche de la collégialité, les arguments ayant motivé la limitation de soins, les thérapeutiques limitées ou arrêtées, les thérapeutiques palliatives mises en place.

\section{Analyse statistique}

Les variables quantitatives étaient exprimées en moyenne ( \pm écart-type $[\mathrm{ET}]$ ) ou médiane (intervalle interquartile). Les variables qualitatives étaient rapportées en nombre et en pourcentage. Les calculs ont été réalisés avec le logiciel Excel (Microsoft, Redmond [Washington] États-Unis).

\section{Résultats}

Durant la période d'étude, le nombre de décès représentait moins de $0,15 \%$ des passages, soit 95 patients au total. Quatre-vingt-onze dossiers ont été inclus. Les quatre patients exclus sont décédés sans qu'aucune décision de limitation n'ait été prise. Il y a eu huit décès en zone d'accueil (9\%), les autres sont survenus en unité d'hospitalisation de courte durée. Parmi les patients inclus, $60 \%$ étaient des femmes. L'âge moyen était de $85 \pm 13$ ans. Le motif d'admission aux urgences était une défaillance d'organe aiguë pour $97 \%$ des patients inclus et, pour $42 \%$, celle-ci rentrait dans l'évolution terminale d'une affection chronique telle qu'une maladie 
neurodégénérative, un cancer évolutif, une insuffisance cardiaque sévère (Tableau 1).

Nous avons pu déduire précisément des données du dossier l'autonomie en calculant le score de Knaus pour 58 patients $(64 \%)$. Parmi eux, près de $86 \%$ des patients $(n=50)$ avaient une autonomie très limitée, soit un Knaus C pour 26 patients et un Knaus D pour 24 patients. Une démarche palliative avait été initiée chez seulement 16 patients, dont dix avaient un projet palliatif connu. La démarche comprenait l'arrêt de la chimiothérapie, la limitation de la ventilation mécanique lors d'une précédente hospitalisation ou encore le fait de ne pas entreprendre une chirurgie pourtant nécessaire. Seulement huit patients avaient désigné une PDC, et deux avaient bénéficié d'un suivi par une équipe de soins palliatifs (SP). L'existence de DA n'était renseignée dans aucun dossier.

D'après la description de l'état de conscience, de l'orientation et du score de Glasgow, suffisamment détaillés dans $74 \%$ des dossiers, le choix éclairé semblait possible chez 15 patients, et parmi ceux-ci, 11 étaient impliqués dans la décision. Dans $83 \%$ des dossiers, il était fait mention d'une démarche pour informer l'entourage, démarche aboutie dans $75 \%$ des cas. L'avis de la famille était connu dans $57 \%$ des cas. Deux familles ont exprimé le souhait d'une réanimation invasive. La collégialité était déduite des dossiers avec le signalement d'un avis téléphonique ou en relevant l'intervention d'un confrère aux urgences ou en préhospitalier. Dans $80 \%$ des cas, la collégialité était recherchée, et le deuxième médecin intervenant était quasi systématiquement (98\%) un médecin extérieur aux urgences et principalement le réanimateur ( $75 \%$ des cas). L'équipe paramédicale n'est sollicitée que trois fois. À trois reprises, la décision venait du patient.

Les arguments de limitation de soins étaient détaillés dans le dossier médical dans $81 \%$ des cas (Tableau 2). Sur les 53 dossiers où le consultant était le réanimateur, c'est celuici qui détaillait la procédure de limitation dans $68 \%$ des cas.

Tableau 1 Principaux antécédents des patients $(n=91)$ retrouvés dans les dossiers

\begin{tabular}{|ll|}
\hline Principaux antécédents des patients & Valeurs (\%) \\
\hline Maladie neurodégénérative & $34 / 76(45)$ \\
Cancer évolutif & $25 / 80(31)$ \\
Insuffisant cardiaque sévère & $18 / 66(27)$ \\
AVC massif & $15 / 69(22)$ \\
Antécédents néphrologiques & $15 / 64(23)$ \\
Insuffisance respiratoire chronique sévère & $14 / 66(21)$ \\
\hline AVC : accident vasculaire cérébral & \\
a Rapport du nombre de patients malades/nombre de patients \\
avec les données disponibles sur les 91 dossiers et pourcentage \\
\hline
\end{tabular}

La traçabilité de la décision de LAT et ses modalités exactes étaient absentes dans $26 \%$ des cas. Le type de thérapeutique arrêtée ou limitée était détaillé dans respectivement 13 et 27 dossiers, soit pour $44 \%$ des patients (Tableau 3). La plupart du temps, il était simplement mentionné « pas de réanimation invasive ». Dans $30 \%(26 / 91)$ des cas, des traitements de réanimation avaient été mis en place. Pour 16 d'entre eux, il s'agissait d'une réanimation d'attente par un remplissage vasculaire seul, sans précision sur son arrêt ou sa poursuite. Des traitements symptomatiques ont été instaurés chez 33 patients (36\%) ainsi que des traitements à visée

Tableau 2 Arguments retrouvés dans 74 dossiers pour la limitation de soins

\begin{tabular}{|ll|}
\hline Arguments pour décision de LAT & $\begin{array}{l}\text { Valeurs } \\
(\mathbf{\%})^{\mathbf{a}}\end{array}$ \\
\hline Terrain/comorbidités & $63(85)$ \\
Irréversibilité de l'affection aiguë à court terme & $54(73)$ \\
Autonomie/qualité de vie future limitée & $41(64)$ \\
Affection sous-jacente incurable et fatale à court & $38(51)$ \\
terme & \\
Absence d'amélioration malgré le traitement actif & $20(27)$ \\
Patient déjà suivi en soins palliatifs & $6(8)$ \\
Risque majeur de dépendance définitive & $3(4)$ \\
aux techniques de suppléance & \\
\hline a Nombre de fois que cet argument est utilisé et pourcentage \\
sur les 74 dossiers avec les données disponibles \\
\hline
\end{tabular}

Tableau 3 Répartition des traitements de réanimation arrêtés au cours de la prise en charge $(n=13)$ et des limitations de soins au début de la prise en charge $(n=27)$

\begin{tabular}{ll|} 
Arrêt thérapeutique & Valeurs $\mathbf{1}^{\mathrm{a}}$ \\
Massage cardiaque externe & $8(68 \%)$ \\
Ventilation mécanique (IOT + VNI) & $9(69 \%)$ \\
Catécholamines & $8(68 \%)$ \\
Limitation de traitement & Valeurs $\mathbf{2}^{\mathrm{b}}$ \\
Pas de réanimation cardiopulmonaire & $3(11 \%)$ \\
Pas d'intubation/ventilation mécanique & $8(30 \%)$ \\
Pas de catécholamines & $3(11 \%)$ \\
Pas de chirurgie & $11(41 \%)$ \\
Pas de dialyse & $2(7 \%)$ \\
Non admis en USIC & $3(11 \%)$ \\
\hline IOT : intubation orotrachéale ; VNI : ventilation non invasive ; \\
USIC : unité de soins intensifs cardiologiques \\
a Thérapeutiques arrêtées au cours de la prise en charge chez \\
13 patients et pourcentage \\
b Thérapeutiques limitées au cours de la prise en charge chez \\
27 patients et pourcentage
\end{tabular}


palliative pour 67 patients (74\%) (Tableau 4). La suspension de l'hydratation, de l'alimentation, des examens complémentaires et de la surveillance était rarement prescrite.

\section{Discussion}

La loi dite Leonetti de 2005, révisée en 2016, fournit un cadre législatif aux situations de fin de vie, et notamment aux modalités de décision de LAT, dont elle souligne l'importance dans la prise en charge des patients. Ces décisions, si elles sont fréquentes dans les services d'accueil des urgences [4], y sont néanmoins difficiles, dans des situations qui nécessitent souvent des prises de décision rapides.

Il parait intéressant de souligner que près de $86 \%$ des patients inclus dans notre étude avaient une autonomie antérieure très limitée (Knaus $\mathrm{C}$ et $\mathrm{D}$ ) et, parmi eux, $40 \%$ étaient grabataires (score de Knaus D). Ces résultats confirment ceux trouvés dans les travaux de Le Conte et al. (33 \%) [9] et de Béreau et al. [10]. Malgré cela, aucun d'entre eux n'avait rédigé de DA, et très peu avaient désigné une PDC. Cette constatation fait ressortir le manque d'anticipation de cette décision par les patients, probablement par méconnaissance, ainsi que l'insuffisance de transmission des informations dans les situations d'urgences. La loi du 2 février 2016 proposait comme solution l'inscription des DA sur la carte vitale du patient, permettant leur consultation dans le cadre de l'urgence.

Il paraît également important de souligner le manque de recours au médecin généraliste $(\mathrm{MG})$ pour les décisions de

\begin{tabular}{|ll|}
\hline $\begin{array}{l}\text { Tableau } 4 \text { Traitements symptomatiques }(n=33) \text { et de confort } \\
(n=67) \text { instaurés }\end{array}$ \\
\hline Traitements instaurés & Valeurs $\mathbf{1}^{\mathrm{a}}$ \\
Antibiotiques & $29(88 \%)$ \\
Traitements cardiotropes & $9(27 \%)$ \\
Antiépileptique & $3(9 \%)$ \\
Aérosols & $1(3 \%)$ \\
Oxygénothérapie & Données non disponibles \\
Thérapeutiques palliatives & Valeurs $\mathbf{2}^{\mathrm{b}}$ \\
entreprises & $64(96 \%)$ \\
Morphine & $40(60 \%)$ \\
Sédation (par midazolam) & $27(40 \%)$ \\
Scopolamine & $2(3 \%)$ \\
Arrêt de l'hydratation & $1(2 \%)$ \\
Arrêt de l'alimentation & \\
\hline a Thérapeutiques symptomatiques instaurées au cours de la prise \\
en charge chez 33 patients et pourcentage \\
b Thérapeutiques palliatives instaurées au cours de la prise en \\
charge chez 67 patients et pourcentage
\end{tabular}

LAT. Alors même que le médecin traitant et/ou les médecins référents devraient être des acteurs centraux de cette décision [11] du fait de leur connaissance globale du patient, de son entourage et de son environnement, ces derniers n'ont quasiment jamais été contactés. Une étude menée en réanimation concernant les décisions de LAT ne montrait une implication du médecin traitant que dans $19 \%$ d'entre elles. Dans cette étude déclarative menée auprès de $\mathrm{MG}$, deux tiers déclaraient ne pas être impliqués dans la décision de LAT, alors même que ces derniers avaient pris des nouvelles de leurs patients dans plus d'un tiers des cas [12]. Ce cloisonnement entre médecine de ville et médecine hospitalière est probablement d'origine multifactorielle (difficultés pour récupérer l'identité de l'interlocuteur, difficulté à joindre le médecin traitant et/ou référent, notamment en dehors des heures ouvrables) et doit nous amener à réfléchir à des pistes d'amélioration de nos pratiques. Il semble important de noter, dans ce travail, le recours dans plus de $80 \%$ des cas à la collégialité par la recherche d'un deuxième avis (le réanimateur dans $75 \%$ des cas), ce qui est en accord avec les résultats observés par Le Conte et al. [4]. Ce résultat est encourageant, alors même que le texte de loi ne paraît pas adapté aux situations d'urgence. Les études évaluant l'applicabilité de cette loi en situation préhospitalière en ont montré de nombreuses limites (défaut d'anamnèse, non-connaissance des stratégies préalablement établies, difficulté à joindre les proches du patient ou son médecin référent) [13]. Ainsi, dans l'étude LATASAMU, $20 \%$ des patients suivis dans le cadre d'une démarche palliative avaient été intubés et hospitalisés en réanimation du fait d'un manque d'informations [14]. Dans l'enquête de Dumont et al. [15], même si la collégialité était plébiscitée par $93 \%$ des médecins interrogés, $49 \%$ avouaient avoir déjà pris seuls une décision de LAT en situation d'urgence. De même, $43 \%$ des médecins urgentistes disaient avoir été confrontés à une prise de décisions de LAT seuls dans l'étude LATASAMU [14] et $12 \%$ des réanimateurs dans l'étude LATAREA [16]. Finalement, dans ces situations particulières non prises en compte par la loi, c'est le concept d'obstination déraisonnable qui prime et dicte la non-instauration de traitements qui ne paraissent pas adaptés. L'équipe paramédicale semblait dans notre étude trop peu impliquée dans les décisions de LAT, alors même qu'elle joue un rôle primordial dans les situations de fin de vie, au contact direct des patients et de leurs familles. Ce faible résultat peut s'expliquer en partie par un manque d'exhaustivité des dossiers médicaux et infirmiers. Avant de recourir à une procédure collégiale de LAT, il convient de recueillir la volonté du patient. S'il n'est pas en l'état de l'exprimer, il est alors recommandé de rechercher les DA, l'avis de la PDC ou en dernier le recours des proches. Dans notre étude, sur les 67 patients dont l'état de conscience était connu, 15 paraissait en état de s'exprimer et, parmi eux, 11 (73\%) ont été impliqués dans la décision. Ce résultat est à 
pondérer par le caractère rétrospectif de l'analyse. La famille était, quant à elle, consultée dans $57 \%$ des cas. Nos résultats sont en accord avec les données de l'étude de Verniolle et al. [17] qui suggéraient la persistance de prises de décisions imparfaites, qu'il s'agisse du manque d'exhaustivité de la consultation des proches, de l'équipe paramédicale ou d'un consultant extérieur comme de la traçabilité. Il est en revanche intéressant de noter que, dans notre étude, les familles des patients étaient informées de la situation critique initiale dans $75 \%$ des cas. Cette démarche des soignants est essentielle à la qualité de prise en charge des situations de LAT. La place de la famille dans l'étude de Le Conte et al. était moins prononcée, avec $60 \%$ de familles prévenues [4] et, dans deux autres études de 2005, elle était équivalente, avec des chiffres respectifs de 72 [18] et $80 \%$ [3].

Notre étude présente plusieurs limites liées au caractère rétrospectif n'incluant que les patients décédés aux urgences, et donc n'incluant pas dans l'analyse les patients décédés dans un autre service. Un biais de recueil du fait de l'analyse rétrospective et un biais de mesure par l'aspect interprétatif des données des dossiers sont également à prendre en compte.

Si les modalités de LAT nous semblent bien respectées, notre méthodologie permet d'identifier un défaut de traçabilité de la procédure. L'intégration dans le dossier des patients d'une page de recueil normalisée à remplir en cas de LAT peut constituer une réponse à ce défaut de traçabilité, permettant de surcroît un rappel pour les équipes sur la démarche à suivre dans cette situation. Ainsi, dans les suites de ce travail, une fiche de ce type a été mise en place dans notre service. Une autre voie d'amélioration des pratiques repose sur la formation. Dans l'enquête préliminaire OPTIDO [19], plus de $80 \%$ des praticiens interrogés avaient estimé que leurs pratiques étaient en adéquation avec les textes, alors que l'évaluation des connaissances a montré que seuls $25 \%$ connaissaient les modalités de la procédure collégiale. En 2011, Matricon et al. [20] avaient montré une assimilation inégale de tous les éléments de la loi Leonetti par les soignants. Les actions de formation ciblées semblent efficaces. Dans une étude prospective, l'introduction d'un support pédagogique, associé à une formation des personnels et à une évaluation des dossiers des patients décédés, permettait d'améliorer la qualité du processus de décision ainsi que sa perception par l'équipe [21]. La mise en place d'un protocole d'aide à la décision éthique aux urgences est également une solution efficace. Dans une étude évaluant ce type d'intervention, la famille et le médecin traitant avaient été informés respectivement dans 93 et $63 \%$ des cas [10]. La Society for Academic Emergency Medicine propose ainsi un modèle de communication pour les situations de fin de vie, fondé sur une succession d'étapes qui permettent d'aboutir à la prise de décision [22].

Enfin, l'amélioration de la prise en charge palliative des patients aux urgences dans les suites d'une LAT semble indispensable. Une thérapeutique palliative avait été entreprise pour $74 \%$ des patients de cette étude. Une étude multicentrique montrait que seuls $50 \%$ des patients décédés avaient bénéficié d'une prise en charge palliative, ce qui paraît insuffisant, la majorité des décès survenant aux urgences ayant été précédés d'une LAT [23]. Une collaboration étroite avec les équipes de SP paraît indispensable. En effet, elle permet une meilleure gestion du décès du patient pour les familles et les aidants [24] et semble permettre une diminution de la durée d'hospitalisation [25]. Dans une revue de la littérature récente, toutes les études pointaient la possibilité de mettre en place des recommandations sur les SP aux urgences. Mais il reste à faire des études plus larges, avec du personnel qualifié pour évaluer l'impact réel que les SP peuvent avoir dans les services d'urgences [26].

\section{Conclusion}

Notre étude met en évidence des axes d'amélioration des décisions de LAT aux urgences. Celle-ci doit concerner la collaboration avec le médecin traitant ou référent, l'information des patients ainsi que la traçabilité. L'apport de la formation et celui d'un cadre protocolisé semblent importants pour fluidifier et simplifier la prise en charge.

Liens d'intérêts : les auteurs déclarent ne pas avoir de lien d'intérêt.

\section{Références}

1. Drees-ministère des Affaires sociales et de la Santé (2014) Le panorama des établissements de santé. Édition 2014. http://www. drees.sante.gouv.fr/le-panorama-des-etablissements-de-sante-edition-2014,11413.html (Dernier accès le 27 décembre 2015)

2. Lalande F, Veber O (2009) La mort à l'hôpital. Insp Generale Aff Soc 11:1-58

3. Tardy B, Viallon A (2005) Fin de vie aux urgences. Réanimation $14: 680-5$

4. Le Conte P, Riochet D, Batard E, et al (2010) Death in emergency departments: a multicenter cross-sectional survey with analysis of withholding and withdrawing life support. Intensive Care Med 36:765-72

5. Le Conte P, Batard E, Pinaud V, et al (2008) Décisions de limitation ou d'arrêt des thérapeutiques actives dans les services d'urgence. Réanimation 17:802-6

6. Tourneret M, Mino JC (2008) An exploratory qualitative study on decisions to reduce current active therapeutic staff working in emergency care and the decisions-making of health care teams in emergency room treatment. Sante Publique 20:517-26

7. Haegy JM, Andronikof M, Thiel MJ, et al (2003) Éthique et urgences : réflexions et recommandations de la Société francophone de médecine d'urgence. J Eur Urgences 16:106-20

8. Blanchet V, Viallard ML, Aubry R (2010) Sédation en médecine palliative : recommandations chez l'adulte et spécificités au domicile et en gériatrie. Med Pal 9:59-70 
9. Le Conte P, Baron D, Trewick D, et al (2004) Withholding and withdrawing life-support therapy in an Emergency Department: prospective survey. Intensive Care Med 30:2216-21

10. Béreau J, Reix A, Faure I, Gabinski C (2013) Mise en place et premières évaluations d'un protocole d'aide à la décision éthique dans un service d'urgence. J Eur Urgences Reanim 25:4-10

11. Ferrand E, Jabre P, Fernandez-Curiel S, et al (2006) Participation of French general practitioners in end-of-life decisions for their hospitalized patients. J Med Ethics 32:683-7

12. Le Meur M, Lainé L, Roucaud N, et al (2014) Implication insuffisante des médecins généralistes lors des décisions de limitation et/ou d'arrêt des traitements au sein d'un service de réanimation. Presse Med 43:e377-e83

13. Jabre P, Combes X, Marty J, et al (2008) Loi $\mathrm{n}^{\mathrm{o}} 2005-370 \mathrm{du}$ 22 avril 2005 relative aux droits des malades et à la fin de vie : application à un cas de médecine préhospitalière. Ann Fr Anesth Reanim 27:934-7

14. Ferrand E, Marty J (2006) Prehospital withholding and withdrawal of life-sustaining treatments. The French LATASAMU Survey. Intensive Care Med 32:1498-505

15. Dumont R, Asehnoune K, Pouplin L, et al (2010) Limitation ou arrêt de thérapeutiques actives en situation d'urgence. Le point de vue des anesthésistes-réanimateurs. Ann Fr Anesth Reanim 29:425-30

16. Ferrand E, Robert R, Ingrand P, et al (2001) Withholding and withdrawal of life support in intensive-care units in France: a prospective survey. Lancet 357:9-14

17. Verniolle M, Brunel E, Olivier M, et al (2011) Évaluation des démarches de limitation et d'arrêt de traitement en salle d'accueil des urgences vitales. Ann Fr Anesth Reanim 30:625-9

18. Le Conte P, Guilbaudeau S, Batard E, et al (2005) Mise en place d'une procédure de limitation ou d'arrêt des soins actifs dans un service d'urgence : évaluation prospective sur 7 mois. J Eur Urgences 18:188-92

19. Neuschwander A, Quesnel C, Fulgencio JP, et al (2010) Décisions de fin de vie et procédure de prélèvement d'organes chez les patients en arrêt de traitement. Connaissances médicolégales et perceptions médicales de réanimation et de soins intensifs. Congrt. Ann Fr Anesth Reanim 29:R236(abstract)

20. Matricon C, Texier G, Mallet D, et al (2013) La loi Leonetti : une loi connue des professionnels de santé hospitaliers? Med Palliat 12:234-42

21. Chastrusse M, Fulgencio JP, Julien F, et al (2012) Loi $n^{\circ} 2005$ 370 du 22 avril 2005 relative aux droits des malades et à la fin de vie : amélioration du processus de décision de limitation et d'arrêt des traitements au moyen d'un programme pédagogique. Étude monocentrique prospective et rétrospective pilote. Presse Med 41:e539-e46

22. Limehouse WE, Feeser VR, Bookman KJ, et al (2012) A model for emergency department end-of-life communications after acute devastating events - part I: decision-making capacity, surrogates, and advance directives. Acad Emerg Med 19:E1068-E72

23. Van Tricht M, Riochet D, Batard E, et al (2012) Palliative care for patients who died in emergency departments: analysis of a multicentre cross-sectional survey. Emerg Med J 29:795-7

24. DeVader TE, Albrecht R, Reiter M (2012) Initiating palliative care in the emergency department. J Emerg Med 43:803-10

25. Delgado-Guay MO, Rodriguez-Nunez A, Shin SH, et al (2015) Characteristics and outcomes of patients with advanced cancer evaluated by a palliative care team at an emergency center. A retrospective study. Support Care Cancer 24:2287-95

26. George N, Phillips E, Zaurova M, et al (2016) Palliative care screening and assessment in the emergency department: a systematic review. J Pain Symptom Manage 51:108-19.e2 